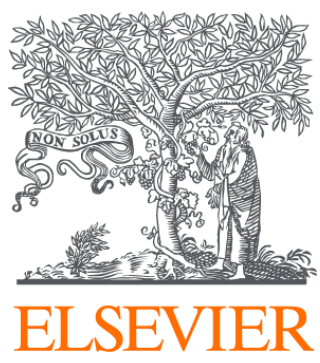

Since January 2020 Elsevier has created a COVID-19 resource centre with free information in English and Mandarin on the novel coronavirus COVID-

19. The COVID-19 resource centre is hosted on Elsevier Connect, the company's public news and information website.

Elsevier hereby grants permission to make all its COVID-19-related research that is available on the COVID-19 resource centre - including this research content - immediately available in PubMed Central and other publicly funded repositories, such as the WHO COVID database with rights for unrestricted research re-use and analyses in any form or by any means with acknowledgement of the original source. These permissions are granted for free by Elsevier for as long as the COVID-19 resource centre remains active. 


\section{Letter to the Editor / Lettre à la rédaction}

\section{Preparing for the Next Pandemic: Lessons Learned from Severe Respiratory Distress Syndrome}

As we approach the sixth anniversary of Severe Respiratory Distress Syndrome (SARS) I wish to add the perspective of a frontline medical leader during the pandemic to the recommendations made by federal and provincial commissions. The lessons learned from events leading up to and during SARS need to be communicated to prepare our hospitals and communities for future respiratory pandemics.

The diagnosis of viral respiratory illness is based on both clinical and radiographic criteria. Preparedness for respiratory pandemics requires optimally functioning radiography equipment as well as the contribution and expertise of radiologists. This should be included among the lessons learned from the Ontario SARS experience.

In 2003 SARS, an airborne viral illness of the corona virus type, spread from China to North America. In Toronto, Ontario, there was a primary phase, as well as a secondary phase during which the infection spread back into the community from hospitalized patients. There have been 2 commissions to assess the Ontario SARS experience. The federal commission (Naylor report) [1] focused on systemwide and public health issues to be addressed when dealing with future pandemics. The provincial commission (SARS Commission Report) by the late Justice Archie Campbell [2] looked at the SARS experience from the provincial and hospital perspectives.

Diseases spread by respiratory transmission within hospitals depends on the professionals within the Medical Imaging Department to provide effective detection and diagnosis of the acute respiratory illness. In addition to disease detection during pandemics, radiologists also can offer specific expertise in risk assessment for health care organizations during and between pandemics.

Radiologists also have expertise in the assessment of issues of quality control and disease monitoring. The funneling of patients through the Imaging Department positions radiologists to identify clusters of complications and diseases that have radiographic manifestations.

Both the Naylor report [1] and SARS Commission reports [2] noted that hospital radiologists raised concerns about the recurrence of SARS weeks before there was formal acknowledgement of a secondary source for the disease. In addition to this important observation by the commissions I believe that there are additional lessons that can be learned from viewing the SARS experience through a radiologist's perspective.

\section{Radiographic Equipment}

Radiography is essential in the detection of respiratory infection during pandemics. The Ontario health care system during the 1980s and 1990s experienced operating deficits. Hospitals and outpatient facilities funded operating deficits at the expense of capital investment, leading to aged radiographic equipment throughout the system. In clinics and hospitals in Ontario in 2003 it was not uncommon to find equipment in use well beyond the usual expected operating life. Such aged equipment was prone to frequent breakdowns as well as diminishing sensitivity to subtle radiographic abnormalities.

The demonstration of subtle pulmonary abnormalities by radiography requires optimally functioning radiography equipment, and the images should be reviewed under ideal conditions. There have been significant technological advances over the past 2 decades, and it is unreasonable that hospital-based physicians should have had to rely on 20-yearold technology as the first line of defense in detecting subtle respiratory infiltrates during a pandemic. Preparedness for pandemics requires regular and timely replacement of radiographic equipment approaching the end of its useful life.

I also note that during both the initial phase (SARS 1) and secondary phase (SARS 2) of the pandemic, computed tomography (CT) of the chest often detected infiltrates in patient with suspected SARS with negative radiographs. This reflected the increased sensitivity of the CT technology compared with the images produced by the radiographic equipment. Planning for future pandemics will need to account for surge capacity in CT imaging to supplement general radiography.

\section{Establishing New Measurable Standards for Radiography}

Radiographic safety and diagnosis depends on a sequence of processes that can be measured using both quantitative or qualitative measures. Measurements need to go beyond the standard Healing Arts Radiation Protection Act (HARP) testing to determine if equipment is clinically adequate. HARP standards for equipment focus primarily on radiation exposure. Measurements need to include additional parameters such as frequency of equipment breakdowns, testing 
using phantoms to assess for subtle radiographic abnormalities, standards for lighting and noise in radiographic reporting areas, assessment of lighting for workstations (or view boxes when used), as well as accuracy and timeliness of radiology transcription.

HARP testing as currently mandated is insufficient to assess the entire process of creating an image and transmitting the report. Therefore, HARP testing is but a sampling of the process affecting patient risk and safety, not a comprehensive assessment of the efficacy and safety of the radiographic images and processes. Whenever a radiologist is concerned about the adequacy of the radiography equipment and images, the fact that the equipment has met the standards for HARP testing may have no bearing on the acceptability of the diagnostic image or the accuracy of the final report,

Preparedness for pandemics should include the establishment of standards for the equipment and processes to achieve the highest sensitivity for subtle infiltrates, achieved at the lowest possible radiation doses, and communicated in the most timely and effective manner.

Provincial standards also should require that the Medical Director of the Imaging Department take responsibility for and sign off on the adequacy of the radiography equipment as well as the entire process and working conditions dealing with the production and interpretation of medial imaging studies. This should become the new normal because the occurrence of pandemics may have short lead times. Therefore, quality concerns of Medical Directors in Medical Imaging Departments about radiography must be addressed proactively, and solving these issues needs to go beyond defaulting to the standards set by the HARP Act.

\section{The Role of the Radiologist and the Chief Radiologist}

The importance of the radiologists in the detection of respiratory airborne diseases in the index hospital was acknowledged by the authors of the federal and provincial reports. The presumptive diagnoses of recurrent SARS made by individual radiologists were transmitted to the attending clinicians verbally and through radiology reports. There was no centralized process to collate and analyse all the radiology reports for new diagnoses of suspected pneumonia. It is recommend that within each hospital, and possibly each region, that there be a centralized system to collate and analyse multiple radiologists' reports for key words or phrases to detect clusters of respiratory disease on wards, or changes in the incidence of respiratory infiltrates within an institution, or even within the community. This is easier to implement where there is an electronic patient record.

There also needs to be a seamless and simple process in which a radiologist can forward specific reports to the Chief Radiologist (Medical Director) and an infection control team for review. This is useful not only during pandemics, but is also of value for the identification of clusters of cases suspicious for more common infectious diseases including influenza and tuberculosis.
The flow of patients and their imaging from throughout the hospital and community through the radiology department gives the Chief Radiologist a unique institutional perspective of risks and disease occurrence throughout the entire hospital. Often a radiologist is able to detect trends and clusters of disease occurring in a hospital well before clinical colleagues.

The concerns of the Chief Radiologist and the radiologists on issues of equipment safety or image quality need to be taken seriously and dealt with in a timely fashion. Few professionals in the hospital have the knowledge and experience of radiologists in the areas of risk assessment arising from deficiencies in the process of creating the medical image and the radiology report. Acknowledgement of and responding to the concerns of medical imaging professionals about the adequacy of equipment technology and processes will ensure that our hospitals and patients will be prepared for respiratory pandemics.

The radiologists, including the Chief Radiologist, should be protected from recrimination if they follow a defined process in reporting their concerns about institutional risk, both specific to medical imaging as well as any risk to patients throughout the entire health care organization [3].

\section{Risk Assessment}

The study of the SARS experience must address the issue of risk assessment. Justice Campbell's [2] report inferred that risk identification and assessment may have been an issue during the second phase of SARS.

The decision makers were dealing with a new disease, of unclear etiology and incubation period, with a large potential population at risk. The disease appeared to arise in clusters, and spread from epicenters.

A radiologist is taught that when a low-level threat could affect large populations then the risk needs to be to dealt with aggressively to reduce it to a minimum level as reasonably achievable for each individual patient at each encounter. Application of this principle during the SARS epidemic should have led to conservative assumptions of incubation times and thus prolonged the use of protective measures for employees and patients well beyond standard known incubation times when dealing with new or unknown infectious entities.

Radiologists also are trained to handle mass casualty events such as occurring from nuclear contamination. In such situations all potential victims are isolated, and aggressive steps are taken to contain the event. Applying this principle to the management of SARS would have led to the aggressive isolation of the hospitals affected during the initial phase of SARS, as well as the strict closure of wards on the suspicion of internal transmission of disease within a hospital.

\section{Recommendations}

The following are recommendations for dealing with future respiratory pandemics. 
- There should be processes to deal with the concerns of radiologists about the safety of imaging equipment and the quality of medical images. Resolution of such issues needs to be transparent and timely.

- Regular testing of radiographic equipment needs to be supplemented by the testing of additional factors including equipment sensitivity, availability, and reliability; the quality of the medical image; the work and safety conditions of technologists, radiologists, and patients; as well as the timeliness and accuracy of transcription.

- The chief radiologist should be required to sign off annually on the adequacy of the radiography equipment as well as the entire imaging process including working conditions involved in the interpretation of studies and the preparation and communication of the report.

- The quality of the inventory of radiographic equipment and technology in hospitals and clinics needs to be reviewed regularly. Replacement must be planned for, independent of anticipated government funding.

- During pandemics radiography may need to be augmented by the use of CT in symptomatic persons with negative radiographs. Planning for pandemics requires planning for surge capacity for CT use.

- The radiology report needs to be in a format that will enable ongoing surveillance and analysis. This would be facilitated by the use of an electronic medical record.

- Radiologists should be included as part of the administrative teams dealing with ongoing surveillance for acute respiratory illness. The Chief Radiologist also needs to be a member of any committees dealing with potential pandemics.

- There should be a whistleblower policy in every health care organization that protects all professionals, including physician leaders, who identify and report suspected risks using established and agreed upon protocols.

The medical and nursing personnel in Ontario during SARS provided exemplary and courageous care during a crisis that was unprecedented in our generation in Canada. Radiologists served a critical role in the detection and management of disease during the pandemic, despite often relying on equipment that has been described as obsolete. To draw on the expertise of radiologists, the radiologist must be part of the organizational decision-making process, and they must have effective equipment at their disposal. The implementation of these recommendations may enhance our ability to recognize clusters of disease during pandemics. These recommendations also may decrease the risk of unrecognized in-hospital transmission and the subsequent secondary propagation of disease back into the community.

Ronn E. A. Goldberg, MD, MBA, FRCPC, DABR Assistant Professor of Medical Imaging (Adjunct) University of Toronto Faculty of Medicine 83 Franmore Circle, Thornhill Toronto, Ontario C4S 3B9, Canada Lecturer, Health Industry Management Program Schulich School of Business MBA Program York University Toronto, Ontario, Canada

E-mail address: ronn.goldberg@ rogers.com

doi:10.1016/j.carj.2009.03.001

\section{References}

[1] http://www.phac-aspc.gc.ca/publicat/sars-sras/naylor/

[2] http://www.sarscommission.ca/.

[3] Goldberg R. The whistleblower in healthcare. Healthc Q 2007;10:10. 